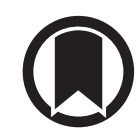

CrossMark

\title{
Why do we still cling to spirometry for assessing small airway function?
}

\author{
To the Editor:
}

With great interest we read the article by ARSHAD et al. [1] and the accompanying editorial [2] in the March 2020 issue of the European Respiratory Journal. We fully agree that early detection and small airway function are both of utmost importance for the management of obstructive lung disease. However, we have some methodological concerns in this context. These should be discussed as certain imitations of the important work at hand.

Since James Hogg's ground-breaking publication in the New England Journal of Medicine, more than 50 years have now passed [3]. Ever since, it has been discussed that considerable destruction must be present in the small airways before it can be detected clinically or with commonly used diagnostic tests $[4,5]$. The latter has not much changed, neither in clinical routine nor research until today. Therefore, the small airways still remain what was previously described as "the lung's quiet zone" [6]. This is important, as the small airways are increasingly recognised to be involved early in the pathogenesis of both bronchial asthma and COPD. Hence, the work by ARSHAD et al. [1] tackles an important topic and potentially provides a link between both entities. We agree that forced expiratory flow at $25-75 \%$ of forced vital capacity $\left(\mathrm{FEF}_{25-75}\right)$ intuitively is more sensitive than parameters of central obstruction, such as forced expiratory volume in $1 \mathrm{~s}$ alone $[1,2]$. However, we want to emphasise that small airway function should not be simply reduced to airway obstruction. In contrast, small airway function comprises heterogenous interactions between ventilation, diffusion, perfusion and inflammation.

Publication of the cross-sectional results from ATLANTIS revealed that small airway function is impaired in over $90 \%$ of asthmatic patients [7]. These changes cannot be addressed by spirometric parameters alone, which must be considered as rather insensitive in general. Recent studies further underline these findings. More advanced techniques, such as multiple breath washout (MBW) testing and oscillometry were demonstrated to be feasible in asthmatic patients. Both add considerable information for the differentiation of asthmatic patients with normal spirometry from non-asthmatic controls, respectively [8]. Likewise, parameters of oscillometry outperformed spirometric parameters for differentiating patients at risk for the development of COPD from healthy controls, as well as from diagnosed COPD patients [9]. Most notably, diagnostic performance of $\mathrm{FEF}_{25-75}$ was just above chance when being used as a single parameter. This also has clinical implications. Lately, oscillometry was shown to be more sensitive than spirometry in identifying bronchodilator response as well as patients with poor asthma control [10].

Oscillometry is recognised as one of the most promising techniques for assessing small airway function in research and clinical practice. This is not only because of the recently demonstrated direct relationship to peripheral obstruction [11]. In contrast to spirometry, oscillometry uses tidal breathing not requiring forced and artificial manoeuvres. This makes it easily applicable, even in early childhood. However, we also see other additional techniques to be valuable in the assessment of small airway function. These include, but are not limited to, well-known conventional tests such as body plethysmography and transfer factor for carbon monoxide (formerly "diffusing capacity"). In addition, more advanced functional techniques such as MBW, capnovolumetry or fractional exhaled nitric oxide are now clinically available. Capnovolumetry seems to be especially interesting for detection of peripheral obstruction in outpatient settings [12], being both inexpensive and easy to perform. Imaging techniques comprise quantitative computed tomography or magnetic resonance imaging. Available data strongly supports the idea of combined approaches. Neither a single test nor even a distinct parameter will ever be sufficient for future

@ERSpublications

Small airway function comprises heterogenous interactions between ventilation, diffusion, perfusion and inflammation that cannot be covered by spirometry https://bit.ly/2LiyQ5E

Cite this article as: Trinkmann F, Watz H, Herth FJF. Why do we still cling to spirometry for assessing small airway function?. Eur Respir J 2020; 56: 2001071 [https://doi.org/10.1183/13993003.01071-2020]. 


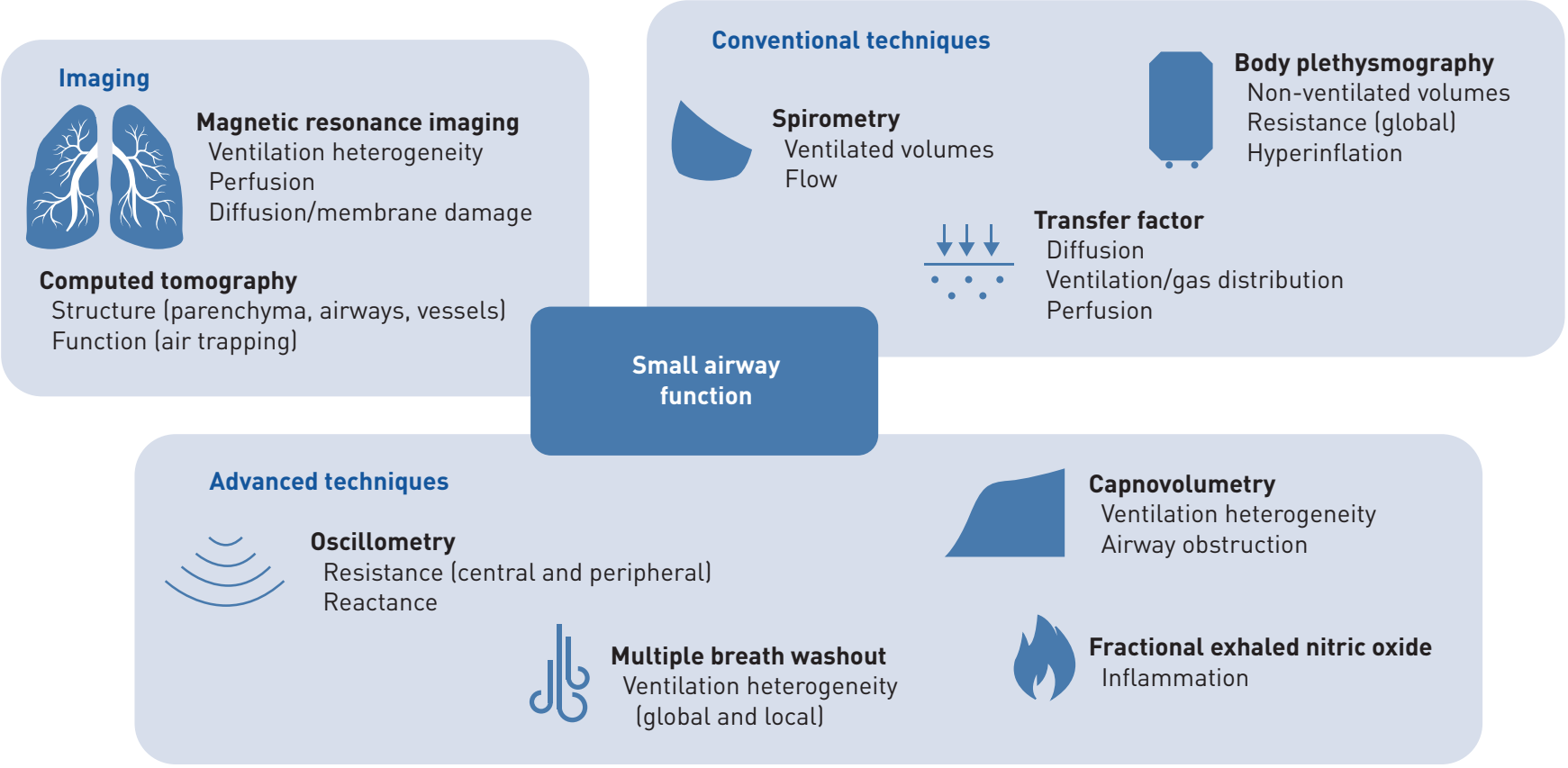

FIGURE 1 Diagnostic tools. Schematic overview of conventional, advanced and imaging techniques for assessment of small airway function with the respective aspects addressed.

assessment of small airway function. The aforementioned techniques provide information about the small airways while addressing different aspects (figure 1).

We believe that it is important to longitudinally assess lung function changes together with risk factors, as proposed in the Isle of Wight Birth Cohort Study. We are aware that today's technology was not readily available at the time of planning the cohort. However, when started today, research should also include more sensitive measures of early changes. There are currently several ongoing multicentre studies investigating both childhood and adult asthma. For example, both the ATLANTIS [7] and ALLIANCE [13] cohorts include measurements of small airway function over time. They will provide additional mechanistic insights into the development and course of disease.

Taken together, those days for spirometry as sole assessment of airway function should be long gone. It is often argued that world-wide availability of other methods is not given, and we want to emphasise that spirometry is not worthless for managing airway disease in general. However, its use for early detection of airway disease is limited. Spirometry should be reasonably extended by techniques aiming, in particular, at small airway function, as they provide additional information. Their final value remains to be determined in future research. However, the availability of such methods must not hinder scientific progress.

Frederik Trinkmann $\oplus^{1,2}$, Henrik Watz ${ }^{3}$ and Felix J.F. Herth ${ }^{1}$

${ }^{1}$ Pneumology and Critical Care Medicine, Thoraxklinik at University Hospital Heidelberg, Translational Lung Research Center Heidelberg (TLRC), Member of German Center for Lung Research (DZL), Heidelberg, Germany. ${ }^{2}$ Dept of Biomedical Informatics of the Heinrich-Lanz-Center, University Medical Center Mannheim, Heidelberg University, Heidelberg, Germany. ${ }^{3}$ Pulmonary Research Institute at LungenClinic Grosshansdorf, Airway Research Center North (ARCN), Member of the German Center for Lung Research (DZL), Grosshansdorf, Germany.

Correspondence: Frederik Trinkmann, Thoraxklinik at University Hospital Heidelberg, Röntgenstraße 1, 69126 Heidelberg, Germany. E-mail: frederik.trinkmann@med.uni-heidelberg.de

Received: 7 April 2020 | Accepted after revision: 6 May 2020

Conflict of interest: F. Trinkmann reports personal fees from Actelion, Berlin Chemie, Boehringer Ingelheim, Chiesi, Novartis, Mundipharma, TEVA, AstraZeneca, Bristol-Myers Squibb, GlaxoSmithKline and Roche, outside the submitted work. H. Watz has nothing to disclose. F.J.F. Herth has nothing to disclose.

\section{References}

1 Arshad SH, Hodgekiss C, Holloway JW, et al. Association of asthma and smoking with lung function impairment in adolescence and early adulthood: the Isle of Wight Birth Cohort Study. Eur Respir J 2020; 55: 1900477. 
2 Polverino F, Soriano JB. Small airways and early origins of COPD: pathobiological and epidemiological considerations. Eur Respir J 2020; 55: 1902457.

3 Hogg JC, Macklem PT, Thurlbeck WM. Site and nature of airway obstruction in chronic obstructive lung disease N Engl J Med 1968; 278: 1355-1360.

4 Hogg JC, Pare PD, Hackett TL. The contribution of small airway obstruction to the pathogenesis of chronic obstructive pulmonary disease. Physiol Rev 2017; 97: 529-552.

5 Stockley JA, Cooper BG, Stockley RA, et al. Small airways disease: time for a revisit? Int J Chron Obstruct Pulmon Dis 2017; 12: 2343-2353.

$6 \quad$ Mead J. The lung's “quiet zone”. N Engl J Med 1970; 282: 1318-1319.

7 Postma DS, Brightling C, Baldi S, et al. Exploring the relevance and extent of small airways dysfunction in asthma (ATLANTIS): baseline data from a prospective cohort study. Lancet Respir Med 2019; 7: 402-416.

8 Trinkmann F, Lenz SA, Schafer J, et al. Feasibility and clinical applications of multiple breath wash-out (MBW) testing using sulphur hexafluoride in adults with bronchial asthma. Sci Rep 2020; 10: 1527.

9 Su ZQ, Guan WJ, Li SY, et al. Significances of spirometry and impulse oscillometry for detecting small airway disorders assessed with endobronchial optical coherence tomography in COPD. Int J Chron Obstruct Pulmon Dis 2018; 13: 3031-3044.

10 Cottee AM, Seccombe LM, Thamrin C, et al. Bronchodilator response assessed by the forced oscillation technique identifies poor asthma control with greater sensitivity than spirometry. Chest 2020; 157: 1435-1441.

11 Foy BH, Soares M, Bordas R, et al. Lung computational models and the role of the small airways in asthma. Am J Respir Crit Care Med 2019; 200: 982-991.

12 Kellerer C, Jankrift N, Jorres RA, et al. Diagnostic accuracy of capnovolumetry for the identification of airway obstruction - results of a diagnostic study in ambulatory care. Respir Res 2019; 20: 92.

13 Fuchs $\mathrm{O}$, Bahmer T, Weckmann M, et al. The all age asthma cohort (ALLIANCE) - from early beginnings to chronic disease: a longitudinal cohort study. BMC Pulm Med 2018; 18: 140.

\section{Assessing small airway function for early detection of lung function impairment}

From the authors:

We thank F. Trinkmann and co-authors for their appreciation of our work and also in raising important questions on the role of small airway function in early detection of lung function impairment associated with asthma and smoking, leading to development of COPD [1]. The focus of our study was not simply on small airway function but rather, the onset and trajectories of lung function impairment associated with asthma and smoking. However, we agree that small airway function is an important part of that assessment. The authors raise two important points in this regard. First, that (at a microscopic level) significant pathology is present in the small airways before it can be detected by commonly used diagnostic tests, and secondly that other tests such as oscillometry, static lung volumes, transfer factor and radiological imaging add to the assessment of small airway function. We agree with both these points. However, we disagree with their assertion that assessment of small airway function using spirometry, and more specifically, forced expiratory flow at $25-75 \%$ of forced vital capacity $\left(\mathrm{FEF}_{25-75}\right)$ is neither useful nor sensitive [2].

The problem with $\mathrm{FEF}_{25-75}$ is not low sensitivity; indeed, it is highly sensitive to changes in small airway function [3]. The problem is large variance, both within and between individuals, where longitudinal follow up of those with impaired $\mathrm{FEF}_{25-75}$ does not consistently correlate with persistent airway obstruction. In older age subjects, changes in $\mathrm{FEF}_{25-75}$ parallel those in forced expiratory volume in $1 \mathrm{~s}$ $\left(\mathrm{FEV}_{1}\right)$ and forced vital capacity (FVC), and as the coefficient of variation is lower in $\mathrm{FEV}_{1}$ and FVC, the $\mathrm{FEV}_{1} / \mathrm{FVC}$ ratio is often used as a marker of airways obstruction [4]. However, whether this was the case for subjects with asthma or smoking exposure during childhood and adolescence is not known. By

@ERSpublications

Both impulse oscillometry and spirometry provide comparable information for small to mid-airway function. However, spirometry is more sensitive in detecting bronchodilator reversibility. https://bit.ly/ 2XYQ0Sa

Cite this article as: Arshad SH, Kurukulaaratchy $\mathrm{R}$, Zhang $\mathrm{H}$, et al. Assessing small airway function for early detection of lung function impairment. Eur Respir J 2020; 56: 2001946 [https://doi.org/10.1183/ 13993003.01946-2020]. 

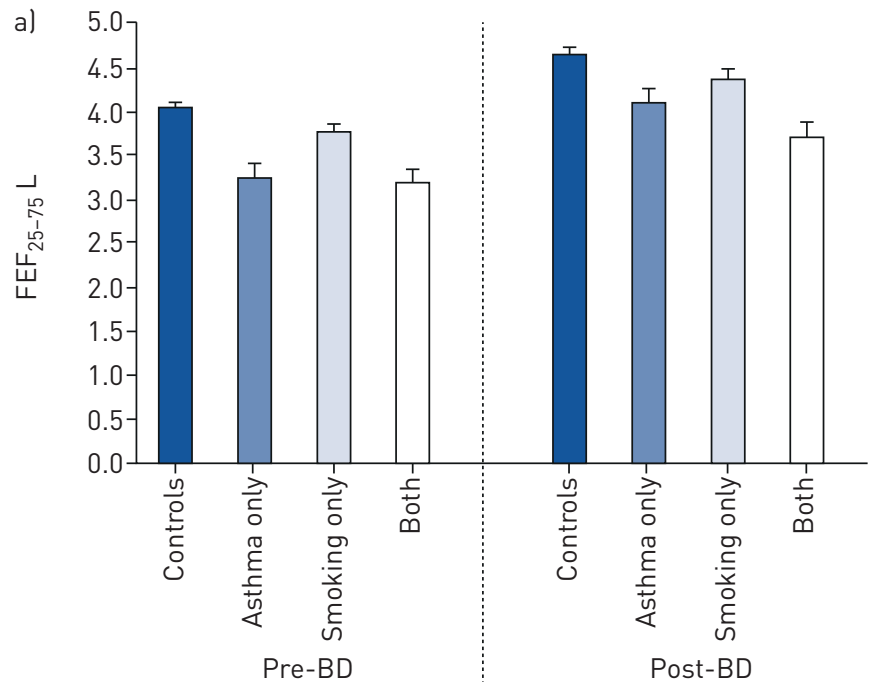

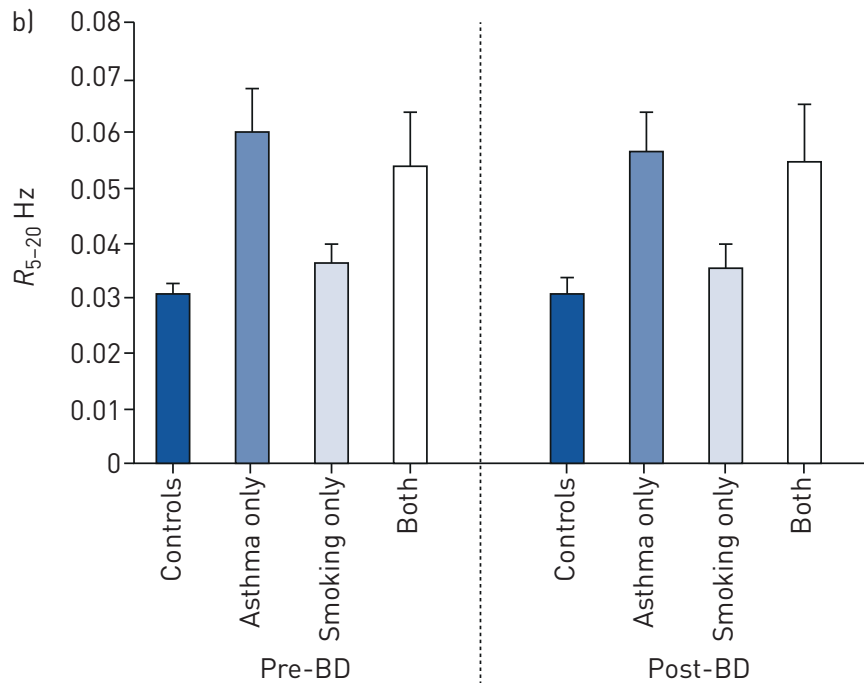

FIGURE 1 The pre-bronchodilator (pre-BD) and post-bronchodilator (post-BD) mean and standard error for each group defined by the presence of asthma and/or smoking at age 26 years: "Controls" (no asthma and did not smoke), "Asthma only" (asthma but did not smoke), "Smoking only" (smoked but did not have asthma) and "Both asthma and smoking" (asthma and did smoke). al Spirometry measured as forced expiratory flow at $25-75 \%$ of forced vital capacity $\left(\mathrm{FEF}_{25-75}\right)$. The pre-BD $\mathrm{FEF}_{25-75}$ was significantly higher in "Controls" compared to all other groups ( $\left.\mathrm{p}<0.001\right)$ and in "smoking" versus "both asthma and smoking" ( $p=0.001)$. Pre-BD asthma was not different to "asthma and smoking"; however, following BD, $\mathrm{FEF}_{25-75}$ improved in asthma patients, such that it was significantly higher than "both asthma and smoking" ( $\left.p=0.005\right)$. b) Oscillometry measured as airway resistance at 5-20 Hz $\left(R_{5-20}\right)$ : the pre-BD $R_{5-20}$ (indicating airway resistance) was significantly lower in "Controls" compared to "asthma" $(p=0.0006)$ and "both asthma and smoking" ( $p=0.0474)$; "asthma" was significantly higher than "smoking" ( $p=0.0198)$. Other comparisons were nonsignificant. Similar patterns were seen for post-BD. All comparisons were carried out using one-way ANOVA and t-test with Bonferroni correction.

longitudinally tracking spirometry, we showed that changes in $\mathrm{FEF}_{25-75}$ parallel $\mathrm{FEV}_{1}$ to some extent but not $\mathrm{FVC}$, given the rapid growth in height during this period. Indeed, $\mathrm{FEF}_{25-75}$ was highly sensitive in detecting signals of airflow limitation before any abnormality was observed in $\mathrm{FEV}_{1}$ or FVC in children aged 10 years when they were stratified with asthma presence at age 26 [1]. Similarly, the first abnormality appearing at age 26 years in only smokers was low $\mathrm{FEF}_{25-75}$, at age 26 years, indicating its usefulness as a marker for early detection of airflow limitation.

F. Trinkmann and co-authors allude to several other modalities that may inform on small airways dysfunction. Body plethysmography and transfer factor for carbon monoxide do require specific equipment and expertise beyond what is easily available in the outpatient consultation setting. The same is true for imaging techniques such as computed tomography (CT) scans and magnetic resonance imaging, with CT scan having the additional disadvantage of the risk of radiation exposure and the technique does not lend itself to large scale screening to detect early COPD. Fractional exhaled nitric oxide is routinely used in the management of asthma, indicating airway inflammation, but it is neither a sensitive nor specific indicator of small airway dysfunction [5]. Longitudinal studies have not confirmed the usefulness of single-breath $\mathrm{N}_{2}$ test in predicting later decline in $\mathrm{FEV}_{1}$ [6]. Further, a large cross-sectional study recently also did not find the newer multiple breath washout to be a sensitive test for small airway dysfunction [7]. These authors concluded that both spirometry and impulse oscillometry were sensitive in defining the presence and severity of small airway dysfunction, are easy to use, and have meaningful association with asthma control and quality of life. We agree that oscillometry is likely to be useful in the management of asthma both in terms of diagnosis, prognosis and control of asthma. We conducted oscillometry in the Isle of Wight birth cohort at age 26 years, albeit not at earlier ages. As this data was not longitudinally available in our cohort, we did not include oscillometry data in our published manuscript. We have now analysed airway resistance at 5-20 Hz $\left(R_{5-20}\right)$, as a measure of respiratory resistance of small to mid-sized airways in our four defined groups (controls, only asthma, only smoking, and both asthma and smoking). Both oscillometry and spirometry $\left(\mathrm{FEF}_{25-75}\right)$ show comparable information (figure 1). However, $\mathrm{FEF}_{25-75}$ was more sensitive for bronchodilator reversibility in asthmatics compared to $R_{5-20}$, with a greater bronchodilator response and higher post- bronchodilator values in those with "asthma" compared to "both asthma and smoking". Further, impulse oscillometry still needs to demonstrate its reliability in predicting progressive lung damage overtime, before its use can be recommended in the diagnosis of early COPD.

Finally, we completely agree that further research is needed in assessing the role of small airway dysfunction in asthma and COPD, utilising longitudinal cohorts with a combination of older and novel techniques to identify at-risk patients early in the course of disease before permanent damage has 
occurred. Studies such as ATLANTIS and ALLIANCE, as mentioned by the authors, will add knowledge in this context $[7,8]$. However, we would like to emphasise the value of birth cohorts with longitudinal data on spirometry and oscillometry, which provide clinically relevant information that can be replicated and used in clinical practice to identify those at risk of COPD. We propose a two-stage approach, which might be more practical and clinically applicable. The first stage should be to perform spirometry and/or oscillometry: both easy to use, inexpensive and widely available tests. Patients showing small airway dysfunction on these commonly available tests can then be further assessed using deeper phenotyping, including body plethysmography, transfer factor and lung imaging.

S. Hasan Arshad ${ }^{1,2,3}$, Ramesh Kurukulaaratchy ${ }^{1,2,3}$, Hongmei Zhang $^{4}$, Claire Hodgekiss ${ }^{1}$, Wilfried Karmaus ${ }^{4}$, John W. Holloway $\oplus^{5}$ and Graham Roberts ${ }^{1,2,3}$

${ }^{1}$ The David Hide Asthma and Allergy Research Centre, Isle of Wight, UK. ${ }^{2}$ Clinical and Experimental Sciences, Faculty of Medicine, University of Southampton, Southampton, UK. ${ }^{3}$ Respiratory Biomedical Centre, University Hospital Southampton, Southampton, UK. ${ }^{4}$ Division of Epidemiology, Biostatistics, and Environmental Health, School of Public Health, University of Memphis, Memphis, TN, USA. ${ }^{5}$ Human Development and Health, Faculty of Medicine, University of Southampton, Southampton, UK.

Correspondence: S. Hasan Arshad, MP: 810, South Block, Southampton General Hospital, Tremona Road, Southampton, UK. E-mail: sha@soton.ac.uk

Received: 22 May 2020 | Accepted after revision: 23 May 2020

Acknowledgement: We would like to acknowledge the help of all the staff at The David Hide Asthma and Allergy Research Centre in undertaking the assessments of the Isle of Wight birth cohort and, specifically, Stephen Potter for helping with lung function data quality checks and preparation of the high quality figure.

Conflict of interest: None declared.

Support statement: The Isle of Wight Birth Cohort assessments have been supported by the National Institutes of Health USA (grant number R01 HL082925), Asthma UK (grant number 364) and the David Hide Asthma and Allergy Research Trust. Funding information for this article has been deposited with the Crossref Funder Registry.

\section{References}

1 Arshad SH, Hodgekiss C, Holloway JW, et al. Association of asthma and smoking with lung function impairment in adolescence and early adulthood: the Isle of Wight Birth Cohort Study. Eur Respir J 2020; 55: 1900477.

2 Hogg JC, Pare PD, Hackett TL. The contribution of small airway obstruction to the pathogenesis of chronic obstructive pulmonary disease. Physiol Rev 2017; 97: 529-552.

3 Walter S, Nancy NR, Collier CR. Changes in the forced expiratory spirogram in young male smokers. Am Rev Respir Dis 1979; 119: 717-724.

4 Detels R, Tashkin DP, Simmons MS, et al. The UCLA population studies of chronic obstructive respiratory disease. 5. Agreement and disagreement of tests in identifying abnormal lung function. Chest 1982; 82: 630-638.

5 Petsky HL, Cates CJ, Kew KM, et al. Tailoring asthma treatment on eosinophilic markers (exhaled nitric oxide or sputum eosinophils): a systematic review and meta-analysis. Thorax 2018; 73: 1110-1119.

6 Buist AS, Vollmer WM, Johnson LR, et al. Does the single-breath $\mathrm{N}_{2}$ test identify the smoker who will develop chronic airflow limitation? Am Rev Respir Dis 1988; 137: 293-301.

7 Postma DS, Brightling C, Baldi S, et al. Exploring the relevance and extent of small airways dysfunction in asthma (ATLANTIS): baseline data from a prospective cohort study. Lancet Respir Med 2019; 7: 402-416.

8 Fuchs O, Bahmer T, Weckmann M, et al. The all age asthma cohort (ALLIANCE) - from early beginnings to chronic disease: a longitudinal cohort study. BMC Pulm Med 2018; 18: 140. 